\title{
Role of Confinement and Surface Affinity on Filling Mechanisms and Sorption Hysteresis of Water in Nanopores
}

\author{
Ezequiel de la Llave, ${ }^{\dagger}$ Valeria Molinero, ${ }^{*,+}$ and Damian A. Scherlis, \\ ${ }^{\dagger}$ Departamento de Química Inorgánica, Analítica y Química Física, Facultad de Ciencias Exactas y Naturales, \\ Universidad de Buenos Aires, Pab II, Ciudad Universitaria, Buenos Aires C1428EHA, Argentina \\ ${ }^{\ddagger}$ Department of Chemistry, University of Utah, 315 South 1400 East, Salt Lake City, Utah 84112-0850, United States
}

ABSTRACT: The liquid-vapor transition in cylindrical pores is studied as a function of pore size and hydrophilicity through molecular dynamics simulations with the $\mathrm{mW}$ coarse-grained model of water. We identify two distinct filling mechanisms, depending on whether the water-pore interaction is smaller or larger than the water-water interaction. In the former case (that we term hydrophobic pore), the formation of the condensed phase proceeds gradually with filling, through the nucleation of a water cluster which grows toward the center of the cavity. In hydrophilic pores, instead, the condensed phase develops in conditions of
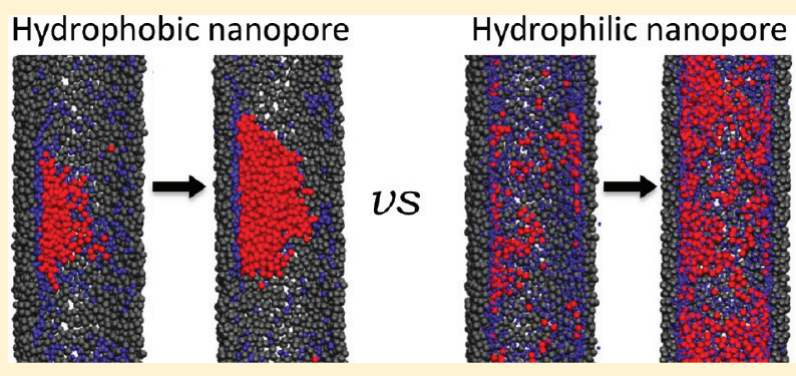
supersaturation, which in principle become more extreme with increasing pore radius and surface affinity. For highly hydrophilic interfaces (those with adsorption energy for water above $10 \mathrm{kcal} / \mathrm{mol}$ ), the equilibrium and dynamical properties of water in confinement turn out to be practically independent of water affinity.

\section{INTRODUCTION}

It is well established that sorption of water and other molecules in ordered mesoporous solids commonly leads to capillary condensation. This phenomenon is characterized by a vertical jump in adsorption isotherms, usually associated with a hysteresis loop, in which features and shape depend on the peculiarities of the pore structure and the temperature. ${ }^{1}$ Hysteresis, from a thermodynamic standpoint, is ascribed to the energetic barriers that must be overcome to nucleate the confined liquid phase from the surface-adsorbed phase during adsorption, and the vapor phase from the liquid during desorption. The microscopic origin of this phenomenon and a description of the adsorption equilibrium, the hysteresis cycle, and its relation to the pore properties are all subjects of active research and matter of restless debate. ${ }^{1-11}$

The first measurements of water sorption in uniform mesoporous materials were focused on MCM-41 matrices, revealing an adsorption pattern characteristic of type $\mathrm{V}$ isotherms, with a pronounced capillary condensation step accompanied by sorptiondesorption hysteresis. ${ }^{3,12}$ A similar behavior was observed for water in FSM-16 silica nanopores. ${ }^{4}$ In both materials, a significant difference between the first and the second sorption runs was informed, both in the low- and high-density branches of the isotherm, presumably originating in the hydroxylation of the pore walls after the first adsorption cycle. ${ }^{4,6,13}$ Grüberg and co-workers proposed two different water-filling mechanisms for mesoporous silica pores with different radius. ${ }^{14}$ According to their NMR analysis, in the narrower pores $(3.3 \mathrm{~nm}$ diameter MCM-41) capillary condensation is preceded by monolayer coverage. In the wider pores ( $8 \mathrm{~nm}$ diameter SBA-15), instead, a radial thickening involving several layers takes place before capillary condensation is observed. Based on sorption calorimetry experiments in MCM-41 silica pores, ${ }^{8}$ Kocherbitov and Alfredsson concluded that capillary condensation is an enthalpydriven event associated with a negative entropic change, suggesting that most of the hydrogen bonds in water are preserved at the conditions of capillary condensation. Specific efforts were carried out to understand the influence of the pore nature on the adsorption-desorption hysteretic phenomenon. In particular, hysteresis was observed to decrease with pore size and to disappear altogether for a diameter situated between 1.4 and $2.0 \mathrm{~nm}$ for water in FSM-16 silica pores. ${ }^{4}$ A similar behavior is also seen for nitrogen, argon, and oxygen, although the pore size at which the hysteresis disappears in these cases is always larger than for water. $^{15-17}$

The study of water confined in nanospaces has not been limited to inorganic oxides of high or moderate hydrophilicity but has been extended to hydrophobic carbon nanopores as well. ${ }^{18-28}$ Different experiments in hydrophobic pores of homogeneous size in the range $0.5-2 \mathrm{~nm}$, reported by Striolo, Kaneko, and others, have shown that water penetrates and is adsorbed by porous carbons. ${ }^{20,22,23,25-28}$ These studies have established that the amount of water adsorbed in these materials is negligible at low pressures, but that filling proceeds with capillary condensation occurring typically at a value of $P / P_{\text {sat }}$ close to 0.5 . Sorption isotherms are characterized by steep jumps and large hysteresis cycles, which disappear above a critical temperature depending on pore width. ${ }^{20,23}$

\footnotetext{
Received: July 11, 2011

Revised: $\quad$ November 7, 2011

Published: December 19, 2011
} 
Complementary to the experimental work, computer simulation has contributed important information to understand the dynamics, equilibrium properties, and phase transitions of water inside the pores. The incidence of the surface chemical nature on the structure and dynamics of $\mathrm{H}_{2} \mathrm{O}$ under confinement was investigated by several authors. Shirono and Daiguji conducted canonical ensemble molecular dynamics and grand canonical Monte Carlo (GCMC) simulations in atomistic models of hydrated silica pores of different diameters. ${ }^{29}$ These authors identified three types of phases, depending on pore size and filling, consisting of (i) a submonolayer coverage with water molecules exclusively solvating the silanol groups, (ii) a condensed monolayer, and (iii) a completely water-filled pore. In addition, they determined that the translational mobility of water in the first adsorption layer was much smaller than the bulk value as a consequence of the strong interaction with the surface groups, in agreement with quasi-elastic neutron-scattering studies confirming that the translational diffusion coefficient of confined water decreases as the pore diameter is diminished. ${ }^{30}$ Romero-Vargas Castrillón and co-workers analyzed the diffusion of water confined between planar surfaces as a function of confinement $^{31}$ and hydrophilicity. ${ }^{32}$ Interestingly, they found that the water diffusion coefficient in the region next to the interface becomes maximum for an intermediate hydrophilicity. This is a consequence of the fact that a hydrophobic surface induces an icelike structure in the interfacial water molecules, while a highly polar interface leads to strong interactions which tend to immobilize the adjacent water layers. ${ }^{32}$ Saugey and collaborators focused on the nucleation of a condensed phase in cylindrical hydrophobic pores using a lattice model in a meanfield approach or with Monte Carlo simulations. ${ }^{33}$ They reported the formation of a spherical liquid droplet for which the nucleation energy barrier was estimated as a function of the chemical potential. Based on a similar mean-field approximation and density functional theory, Monson reproduced and discussed the shape of water adsorption isotherms in porous carbon. ${ }^{34}$ The effect of different water-substrate interaction strengths on the phase states of water was investigated by Brovchenko et al. using Gibbs ensemble Monte Carlo simulations in cylindrical and slitlike nanopores with featureless surfaces. ${ }^{35}$ These authors observed bulklike liquid-vapor phase transitions in hydrophobic pores, whereas they identified three additional types of phase coexistence in more hydrophilic systems and classified them as first layering transition, second layering transition, and prewetting. Liquid coexistence with an adsorbed bilayer was typically seen for the most hydrophilic pores. To the best of our knowledge, this is the only computational study systematically exploring the role of surface affinity on the vapor-liquid phase transition of water in nanoscopic channels.

The simulation of water in hydrophobic graphitic pores has been conducted by several authors, in particular by the groups of Striolo and of Kaneko, employing grand canonical Monte Carlo techniques. $^{20,21,23-26}$ Computational results have accurately reproduced the experimental adsorption isotherms and hysteretic loops. ${ }^{23,25,26}$ Based on simulations, Kaneko and coauthors have proposed that the mechanism of water filling of carbon nanopores involves the formation of $\mathrm{H}_{2} \mathrm{O}$ clusters exhibiting a "hydrophilic to hydrophobic transformation", 24 among which octamers, nonamers, and decamers play a key role in allowing adsorption in pores of $1 \mathrm{~nm}$ and wider. ${ }^{23-26}$ Striolo and coworkers have examined the effect of carbonyl functionalization of carbon pores, analyzing the incidence of the density and the distribution of the oxygen decoration on the water sorption isotherms. $^{21}$

In recent work, ${ }^{9}$ we have shown that for water in moderately hydrophilic pores, with adsorption energies comparable to those found in MCM-41 and FSM-16 silica pores, there is an onset filling at which capillary condensation is unleashed, forming a condensed liquid phase which coexists with a low-density phase consisting of water adsorbed on the pore walls. Above this water content, further addition of molecules to the system does not alter the densities of the two phases in equilibrium but causes an increase in the amount of the condensed liquid phase. We found that in $3 \mathrm{~nm}$ pores, the surface density just before the point of capillary condensation exceeds by a factor of 2 the density in equilibrium after condensation; i.e., the phase transition occurs in conditions of supersaturation. This behavior, which can be related to the hysteresis cycle in adsorption-desorption isotherms, is not observed in a narrower pore. In the present study, we focus on the influence of the diameter and the hydrophilicity of the nanopore on the liquid-vapor transition in cylindrical pores. In particular, we examine the mechanisms and the water contents leading to condensation and analyze their role in the hysteresis cycle. To this end, we consider three different pore sizes (15, 30, and $40 \AA$ in diameter) and explore the effect of pore-water interaction energies ranging from ca. 6 to $14 \mathrm{kcal} / \mathrm{mol}$. This range encompasses the heats of adsorption observed for dehydroxylated (hydrophobic) to highly hydroxylated (hydrophilic) silica matrices. $6,12,13,36,37$

\section{METHODS}

A. Water Force Field. The interaction between water molecules was described by the coarse-grained $\mathrm{mW}$ water model, ${ }^{38}$ which represents each molecule as a single particle interacting through anisotropic short-ranged potentials that encourage "hydrogen-bonded" water structures. The mW model uses the short-ranged interaction form of the Stillinger-Weber (SW) potential $^{39}$ which consists of a sum of two-body attraction terms $\left(\phi_{2}\right)$, which favor high coordination, and three-body repulsion terms $\left(\phi_{3}\right)$, which reinforce tetrahedral "hydrogen-bonded" configurations:

$$
\begin{aligned}
& E=\sum_{i} \sum_{j>i} \phi_{2}\left(r_{i j}\right)+\sum_{i} \sum_{j \neq i} \sum_{k>j} \phi_{3}\left(r_{i j}, r_{i k}, \theta_{i j k}\right), \\
& \phi_{2}\left(r_{i j}\right)=A \varepsilon\left[B\left(\frac{\sigma}{r_{i j}}\right)^{4}-1\right] \exp \left(\frac{\sigma}{r_{i j}-a \sigma}\right), \\
& \phi_{3}\left(r_{i j}, r_{i k}, \theta_{i j k}\right)=\lambda \varepsilon\left[\cos \theta_{i j k}-\cos \theta_{0}\right]^{2} \exp \left(\frac{\gamma \sigma}{r_{i j}-a \sigma}\right) \\
& \times \exp \left(\frac{\gamma \sigma}{r_{i k}-a \sigma}\right)
\end{aligned}
$$

where $r_{i j}$ is the distance between particles $i$ and $j, q_{i j k}$ is the angle formed by the vectors between the positions of the $i-j$ and $i-k$ pairs of particles, $A=7.049556277, B=0.6022245584, p=4$, $q=0, \gamma=1.2, a=1.8$, and $\theta_{\mathrm{o}}=109.47^{\circ}$. The value of the parameter $\lambda=23.15$ dictates the strength of the tetrahedral interactions, while $\sigma=2.3925 \AA$ and $\varepsilon=6.189 \mathrm{kcal} / \mathrm{mol}$ modulate the characteristic length and strength of water-water interaction, respectively. A point worthy of mention is that although the $\mathrm{mW}$ model does not include electrostatic terms or explicit hydrogen 
atoms, it is able to accurately reproduce the phase behavior, anomalies, and structure of solid and liquid water, of low-density amorphous ice, and of clathrates, and it has been successfully employed to elucidate the nucleation and growth of ice in bulk and under confinement. ${ }^{38,40-43}$

B. Model of the Pore. The structure of the pore walls is amorphous, having been taken from an instantaneous configuration of liquid water simulated at $298 \mathrm{~K}$ and $1 \mathrm{~atm}$. Cylindrical channels of 15,30 , and $40 \AA$ diameter were built carving out blocks of water, to produce nanopores of length 200,100, and $150 \AA$, respectively. When filled, such systems involve a total number of water molecules ranging from 2900 to 8500 depending on size and filling percentage. The pore walls are always wider than $1 \mathrm{~nm}$, well beyond the $4.32 \AA$ cutoff of the $\mathrm{mW}$ force field. The particles compounding the pore walls are described by the potential in eq 1, but adopting different values for $\sigma$ and $\varepsilon$ to modulate the interactions with the water molecules contained within. In addition, the molecules integrating the wall interact with their first neighbors through soft harmonic potentials, which preserve the overall structure of the matrix while allowing for vibrations around the corresponding equilibrium positions. To prevent infiltration of liquid water through the pore structure, $\sigma$ was set to $2.8 \AA$ for the cross interaction between the particles of the pore wall and the water molecules. To explore the effect of surface hydrophilicity, the value of $\varepsilon$ associated with the waterpore interaction was systematically spanned in the range from 5.5 to $10 \mathrm{kcal} / \mathrm{mol}$ (while $\varepsilon=6.189 \mathrm{kcal} / \mathrm{mol}$ for the waterwater interaction). The adsorption energy corresponding to each value of $\varepsilon$ was determined from the average interaction energy of water with the pore wall obtained in molecular dynamics runs with water molecules that do interact with the pore wall but not with each other, as previously described in ref 9 . The selected values of $\varepsilon$ produce an interval of adsorption energies extending from 6.1 to $13.7 \mathrm{kcal} / \mathrm{mol}$.

C. Simulation Procedure. Molecular dynamics simulations were performed in the canonical (NVT) ensemble with cubic periodic boundary conditions, using the massively parallel LAMMPS code. ${ }^{44}$ The equations of motion were integrated using the velocity Verlet algorithm with a time step of $5 \mathrm{fs}$, applying the Nose-Hoover thermostat with a relaxation time of 0.5 ps to control the temperature. To produce a uniform distribution of water along the pore, all runs were subject to an initial equilibration time of $10 \mathrm{~ns}$ at high temperature $(600 \mathrm{~K}$ for the widest pore and $400 \mathrm{~K}$ for the others). This stage was followed by a cooling ramp of $2 \mathrm{~K} \mathrm{~ns}^{-1}$ down to $298 \mathrm{~K}$. Thereafter, statistical sampling was prolonged for $25 \mathrm{~ns}$. For each pore size and affinity, simulations were conducted at different filling percentages, to determine the point of capillary condensation.

\section{RESULTS AND DISCUSSION}

A. Filling Mechanisms. It is possible to distinguish between three contrasting pore-filling behaviors, according to the radius and hydrophilicity. Along this work, we will consider that a pore is hydrophobic or hydrophilic depending on whether the water-wall interaction is weaker or stronger, respectively, than the water-water interaction (the absorption energy of water on a pore with waterlike interactions is $6.83 \pm 0.08 \mathrm{kcal} / \mathrm{mol}$ at $\left.298 \mathrm{~K}^{9}\right)$. The simulations indicate that the filling of the narrowest pore ( $15 \AA$ diameter) is basically independent from the watersurface affinity, and the condensation of the liquid plug occurs
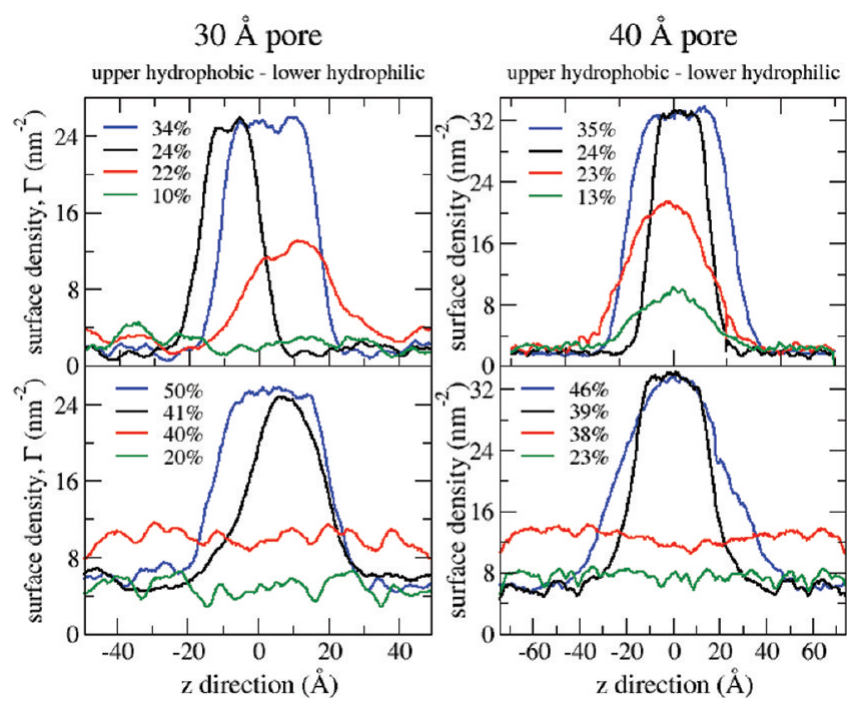

Figure 1. Water surface density profiles inside 30 and $40 \AA$ pores as functions of the axial coordinate, for different filling fractions before and after condensation. The adsorption energies of water are 6.1 and $8.5 \mathrm{kcal} / \mathrm{mol}$ for the hydrophobic (upper panels) and hydrophilic (lower panels) nanopores.

close to the equilibrium surface density of water. In the wider pores, instead, two different regimes can be characterized.

For the $15 \AA$ diameter pore, the growth of a water monolayer is seen with the progressive addition of water molecules, until the surface density reaches a certain value $\Gamma_{\mathrm{eq}}$ and condensation takes place. After this event, two phases coexist in equilibrium: (i) a lowdensity phase of molecules adsorbed at the interface whose surface density is $\Gamma_{\text {eq }}$ and (ii) a higher density phase forming a liquid plug. As it will be discussed in more detail in the next section, the increase of the water-wall interaction gives rise to denser surface-adsorbed water layers in equilibrium with the condensed liquid phase, without altering the density of the latter or the filling mechanism. Due to the small radius of the cavity, any further thickening of the adsorbed layer above $\Gamma_{\text {eq }}$ would initiate the formation of the plug. Once the adsorbed layer reaches the equilibrium surface density, a minor fluctuation is enough to bridge the gap between the pore walls. We find that this process is qualitatively the same for all the water-wall interaction strengths explored.

A richer picture emerges from the analysis of the wider pores, for which we identify two different filling mechanisms, corresponding to the hydrophobic and hydrophilic regimes. Figure 1 displays the average surface density of water molecules along the axial direction in the 30 and $40 \AA$ pores, for two different water-surface interaction strengths. The upper and lower panels present, respectively, the typical results for a hydrophobic and a hydrophilic interface (the corresponding adsorption energies are 6.1 and $8.5 \mathrm{kcal} / \mathrm{mol})$. The data was collected over $25 \mathrm{~ns}$ trajectories equilibrated at the indicated water contents. In highly hydrophilic pores, the density profiles show a homogeneous growth along the pore axis, developing a surface-adsorbed water multilayer until the capillary condensation occurs. This is clearly seen in the lower panels of Figure 1: for fillings of up to ca. $40 \%$ (38\% in the case of the $40 \AA$ pore), the trend to maximize the interactions with the walls leads to a uniformly distributed water layer along the pore. There is a sharp transition once the plug is formed: the density of the surface adsorbed layer drops to its equilibrium value $\Gamma_{\mathrm{eq}}$ which depends on the hydrophilicity of the wall. Beyond that point of 

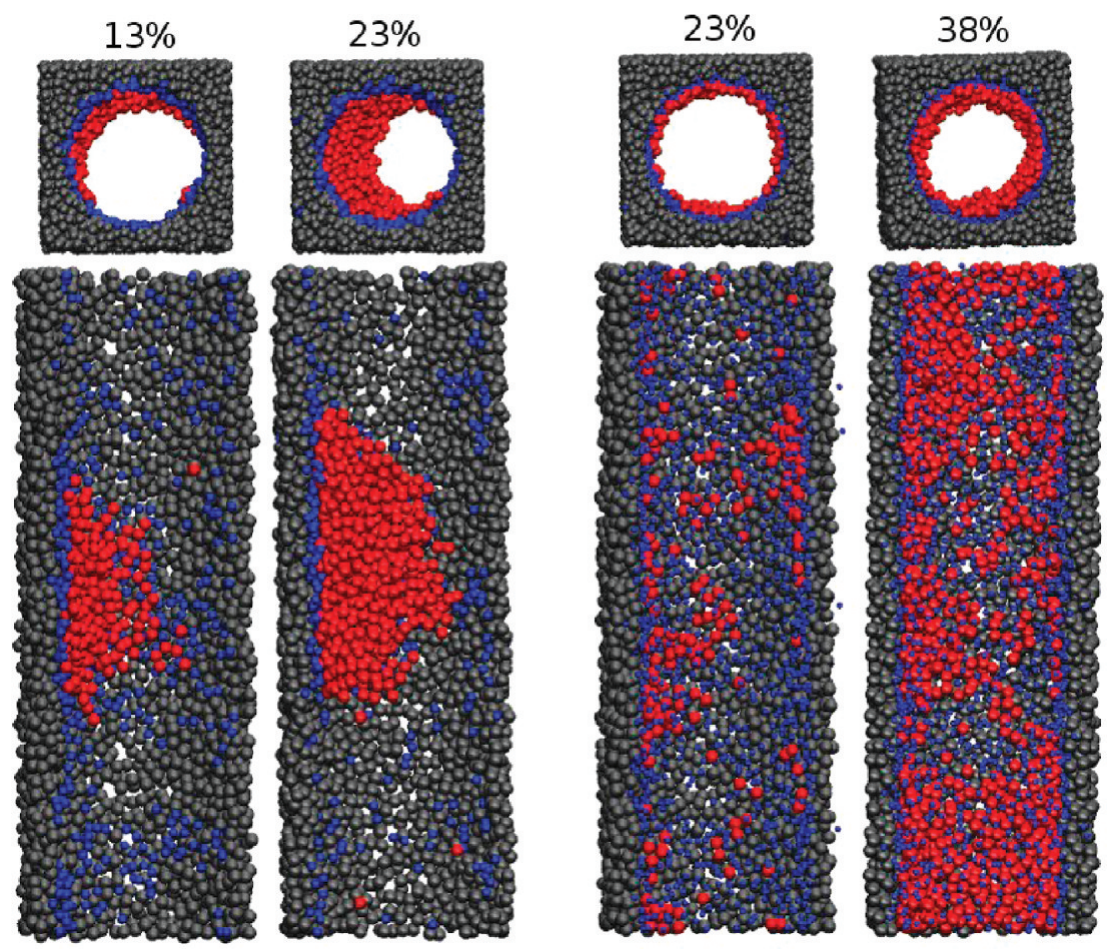

Figure 2. Snapshots of the hydrophobic (left) and hydrophilic (right) $40 \AA$ pore, representing fillings below the point of plug formation. In the hydrophobic system there is a localized accumulation of water, at variance with the hydrophilic pore, for which the water layer grows homogeneously. For better appreciation of the aggregate, the water particles sitting within $16 \AA$ from the center of the pore are red-colored, while the rest of the water is shown in blue. Particles belonging to the pore walls are shown in gray. Different views are presented, perpendicular and parallel to the pore axis.

capillary condensation, the two phases (surface-adsorbed and liquid plug) coexist and any further addition of water only produces the growth of the plug without significantly affecting the densities of these two phases.

On the other hand, the upper panels of Figure 1 reveal a quite different behavior corresponding to a hydrophobic surface. Here the rise in water content is reflected in a localized increase of the surface density. The water layer thickens inhomogeneously, nucleating a water droplet that appears to be in equilibrium with a surface-adsorbed water layer whose density remains practically unchanged during the filling process. This droplet is reminiscent of the asymmetric vapor bubble predicted by Saugey et al. on the basis of a simple lattice model. ${ }^{33}$ The local accumulation of water in a particular region of the pore continues until the size of the liquid droplet closes the gap between opposite sides of the pore wall leading to the gradual formation of a liquid plug. Figure 2 illustrates the distribution of water in both the hydrophobic and hydrophilic nanopores at filling fractions below the ones that lead in each case to the full formation of the water plug. Note that in the hydrophobic cavity, the accumulation of water molecules is also inhomogeneous around the circumference of the pore.

It should be noticed that all pores examined in this study, including those considered hydrophobic, exhibit a water affinity at least three times higher than that corresponding to graphene and carbon nanotubes, where the interaction energy with the surface is around $2 \mathrm{kcal} / \mathrm{mol} .{ }^{21,24}$ The water adsorption before condensation reported for carbons is significantly less than the uptake informed here and follows a different mechanism. ${ }^{20-26}$ The water molecules are not uniformly distributed along the surface but form aggregates of several molecules that, once a critical cluster size is reached, coalesce to produce the condensed phase. ${ }^{23-26}$
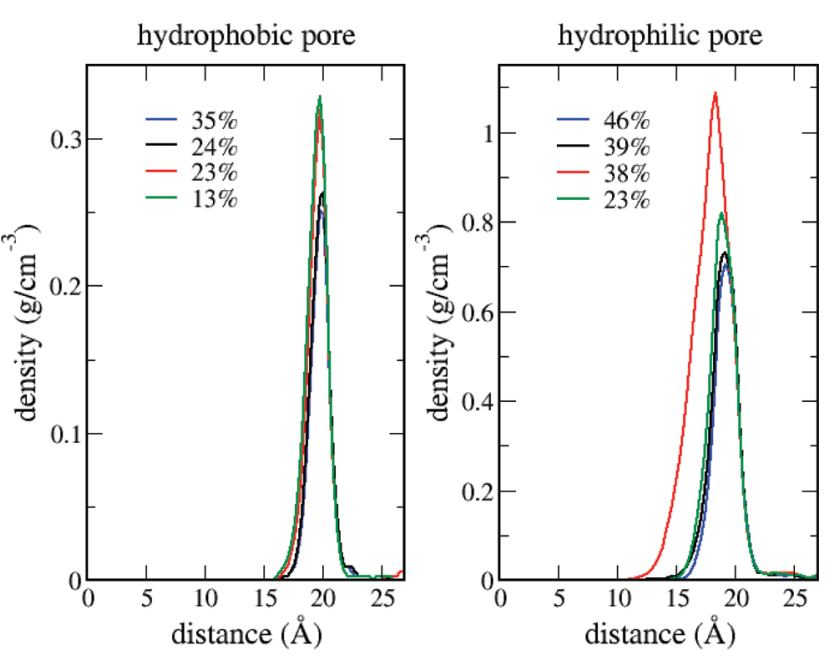

Figure 3. Radial density profiles of water in the $40 \AA$ pore for various filling percentages. Left: hydrophobic (adsorption energy $6.1 \mathrm{kcal} / \mathrm{mol}$ ). Right: hydrophilic (adsorption energy $8.5 \mathrm{kcal} / \mathrm{mol}$ ).

Figure 3 depicts the radial density profiles of the surfaceadsorbed phase as a function of the distance to the center of the pore. The curves represent various filling conditions before and after condensation, averaged in the region excluding the condensed phase (liquid plug or its droplet precursor) for the $40 \AA$ pore. The amorphous structure of the wall smoothes out the radial profiles which result in a single peak for both the hydrophobic and hydrophilic systems, at variance with the characteristic multilayer profiles templated by more ordered or uniform surfaces exhibiting a well-defined peak for each layer (see, for 

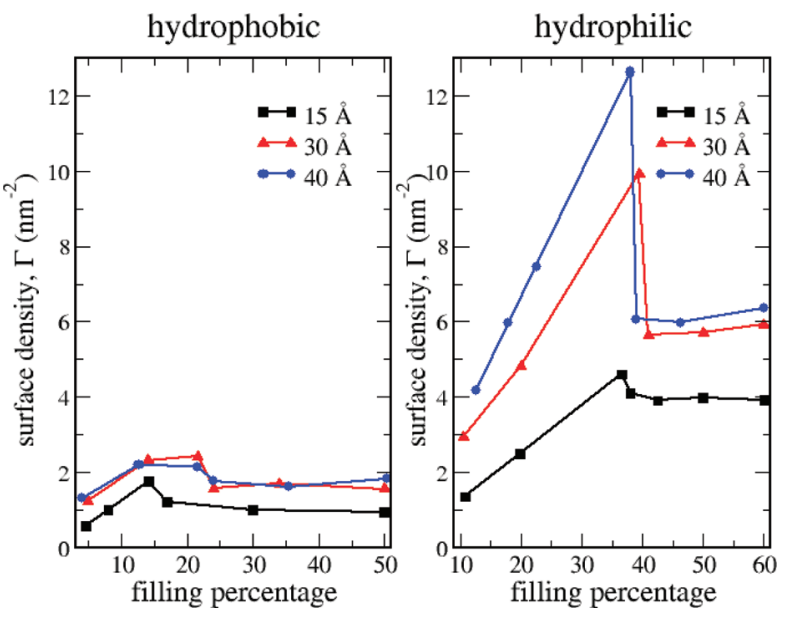

Figure 4. Water surface density as a function of filling, for hydrophobic (left) and hydrophilic (right) systems of different diameters. Surface density values are averaged on the regions excluding the liquid droplet or plug, when present.

example, refs 2 or 42). The green and red curves in Figure 3 represent filling percentages prior to full plug formation, which have been chosen in coincidence with those depicted in Figure 2. In the hydrophobic case, the profiles, which exclude the region corresponding to the droplet, clearly show that the density of the surface-adsorbed phase does not vary appreciably with water content. It is possible to detect a small decrease in the density of the surface-adsorbed phase right after a full plug is formed, suggesting that the system undergoes a very soft transition after all, at a filling of $23 \%$. In the hydrophilic pore, instead, there is a gradual increase of the surface density until the sharp transition associated with the appearance of the condensed phase. The black and blue curves illustrate that above the onset filling fraction, at which the liquid plug forms, the equilibrium surface density is hardly affected by the water content.

B. The Hysteresis Phenomenon. An interesting feature evinced in the radial profiles shown in Figure 3 is the dependence of the equilibrium surface density on the water affinity of the interface. The surface-adsorbed phase in the hydrophilic pore with adsorption energy $8.5 \mathrm{kcal} / \mathrm{mol}$ is almost three times denser than in the hydrophobic pore with adsorption energy $6.1 \mathrm{kcal} / \mathrm{mol}$. This finding is directly related to the hysteresis phenomenon and is addressed in what follows.

In order to characterize the onset filling $f_{\text {onset }}$ at which capillary condensation is observed, molecular dynamics simulations were conducted for different amounts of water in the pore, checking for the appearance of a condensed phase over 25 ns long trajectories. These systematic inspections were narrowed down to determine $f_{\text {onset }}$ with an uncertainty of $1-2 \%$.

Before capillary condensation, and provided the distribution of molecules is uniform along the axial direction (which is not the case for the less attractive pores), the surface density increases linearly with the filling percentage $f$, as dictated by the purely geometrical relation

$$
\Gamma=\frac{N}{A}=\frac{f V \rho}{100 A}=\frac{f \pi R^{2} L \rho}{2 \pi R L 100}=\frac{\rho}{2} \frac{f}{100} R
$$

where $\Gamma$ is the surface density of water, $V$ is the pore volume, $A$ is its area, $N$ is the number of water molecules contained in it, $f / 100$ is the filled volume fraction, and $\rho$ is the number density of liquid

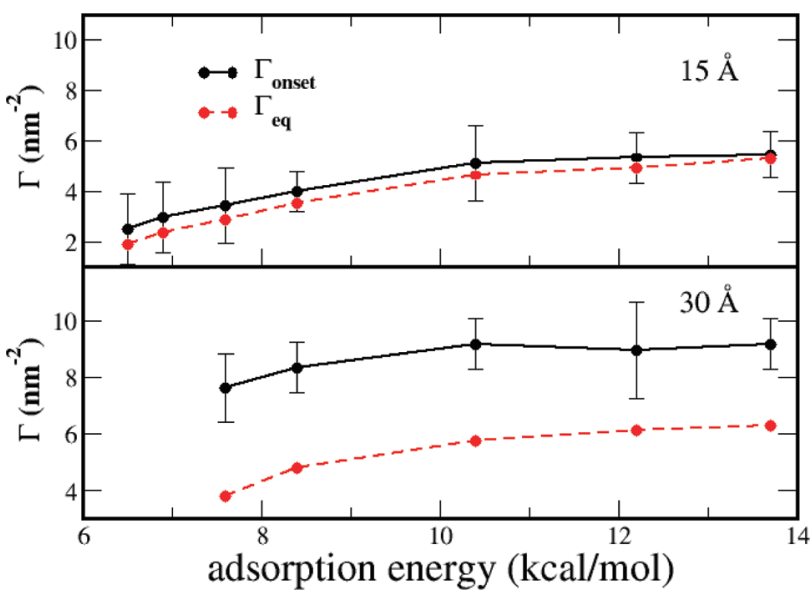

Figure 5. Equilibrium $\left(\Gamma_{\text {eq }}\right)$ and onset $\left(\Gamma_{\text {onset }}\right)$ surface density of water versus adsorption energy. Upper panel: $15 \AA$ pore. Lower panel: $30 \AA$ pore. $\Gamma_{\text {eq }}$ is the surface density observed to be in equilibrium with the condensed phase. $\Gamma_{\text {onset }}$ is the surface density computed from $f_{\text {onset }}$, using eq 2 (see text).

water. Figure 4 depicts the surface density of the suface-adsorbed phase as a function of filling, for pores of different diameters with hydrophilic and hydrophobic water-wall interactions. These densities have been averaged on the region excluding the liquid plug or the droplet. Two important results are evident from Figure 4: (i) the degree of supersaturation before capillary condensation in the hydrophilic channels strongly depends on the pore radius, while it is almost radii independent in the hydrophobic pores, and (ii) the surface density of the adsorbed phase in equilibrium with the liquid, $\Gamma_{\text {eq }}$ does not appear to have a significant dependence on the radius but is markedly affected by hydrophilicity. The effect of pore radius on supersaturation in hydrophilic pores has been already discussed in our previous study; ${ }^{9}$ here, we see it is still valid through a broader range of affinities and pore radii. The excess density needed to nucleate the condensed phase is indicative of hysteresis in capillary condensation. A supersaturation of the surface is required to develop the liquid out of equilibrium, and this overshooting of the density must be more pronounced as the pore becomes wider. As has been shown in ref 8 , the formation of the plug in the hydrophilic nanopores is triggered by a fluctuation that allows to bridge the gap across the channel. In the hydrophobic pore, on the contrary, the condensed liquid phase builds up gradually as a droplet attached to the surface.

With regard to the second result, the data in Figure 4 indicate that the equilibrium surface-adsorbed density $\Gamma_{\text {eq }}$ seems to converge for diameters above $3 \mathrm{~nm}$. For the hydrophobic system, the equilibrium surface density is roughly $1 \mathrm{~nm}^{-2}$ in the $15 \AA$ diameter pore and $1.5 \mathrm{~nm}^{-2}$ in the 30 and $40 \AA$ diameter pores. In the hydrophilic case, $\Gamma_{\text {eq }}$ has an average value of about $4 \mathrm{~nm}^{-2}$ in the smallest pore and $6 \mathrm{~nm}^{-2}$ in the two larger channels. This suggests that, for a given water-wall interaction, the curvature of the pore modulates the equilibrium density of the surfaceadsorbed phase. In the narrow nanotube, a density of, say, $1.5 \mathrm{~nm}^{-2}$ involves a more repulsive situation than in the larger pores. As the curvature is relaxed and the flat limit is approached, $\Gamma_{\text {eq }}$ becomes independent of the pore radius. In both the hydrophilic and hydrophobic pores, this limit has been basically reached already for a diameter of $3 \mathrm{~nm}$. 


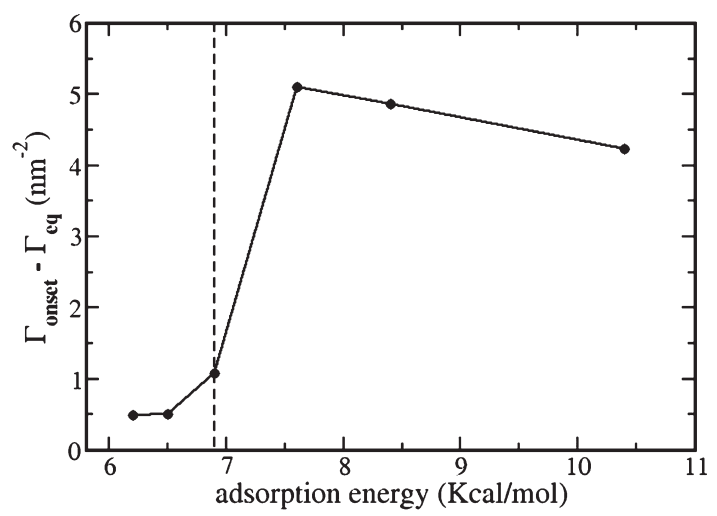

Figure 6. Difference between the surface density at the onset of capillary condensation ( $\Gamma^{*}$ onset or $\Gamma_{\text {onset }}$ depending on hydrophilicity, see text) and the surface density in equilibrium $\left(\Gamma_{\text {eq }}\right)$ as a function of the absorption energy for water in the $40 \AA$ pore. The dashed vertical line indicates the adsorption energy for a water-pore interaction identical to water-water interaction.

The value of $\Gamma_{\text {eq }}$ is instead highly sensitive to the water affinity of the interface. Comparison of the left and right panels of Figure 4 reveals that a rise of $40 \%$ in the adsorption energy produces a $300 \%$ increase in the equilibrium density. This dependence is presented in Figure 5 for the pores of 1.5 and $3 \mathrm{~nm}$, for the full range of interaction energies explored, with the exception of the most hydrophobic data points in the wider pore (for which the droplet develops continuously and an onset filling cannot be established). The increment in $\Gamma_{\text {eq }}$ can be understood noticing that a higher strength of the water-surface interaction potential $\varepsilon$ parameter causes a decrease in the chemical potential of the adsorbed phase, without significantly affecting the chemical potential of the condensed phase. For the larger interaction energies, above $10 \mathrm{kcal} / \mathrm{mol}$, the value of $\Gamma_{\text {eq }}$ seems to plateau at a value that is consistent with a complete monolayer. Once the water molecules saturate the interface, the effect of a further increase of $\varepsilon$ on the chemical potential of the adsorbed phase becomes less and less important. In other words, for coverages higher than a monolayer, the hydrophilic walls are fully carpeted with water molecules and the solid will therefore behave more or less like a water-made pore, regardless of the value of $\varepsilon$. As a matter of fact, $\Gamma_{\text {eq }}$ varies slowly once it has reached a value of $4 \mathrm{~nm}^{-1}$, to eventually stabilize at around $6 \mathrm{~nm}^{-1}$, which roughly corresponds to an intermediate coverage between a water monolayer and a bilayer.

Figure 5 also shows the onset surface density $\Gamma_{\text {onset }}$ i.e., the density corresponding to the onset filling $f_{\text {onset }}$ occurring just before the point of capillary condensation. These two quantities are related by eq 2 in the hydrophilic pores, where the surfaceadsorbed phase is axially homogeneous. Interestingly enough, the value of $\Gamma_{\text {onset }}$ increases at a similar rate as the equilibrium surface density. Indeed, the difference $\Gamma_{\text {onset }}-\Gamma_{\text {eq }}$ remains practically constant for the hydrophilic range of interaction energies. As pointed out before, this difference is a measure of the supersaturation from the equilibrium surface density and can be associated with the degree of hysteresis in the corresponding adsorption-desorption isotherm. Since we cannot quantitatively relate in a simple manner the surface density (or filling percentage) with pressure, we cannot assess precisely the extension and shape of the hysteretic loop. However, we can conclude that water affinity does not play a role in the hysteresis as significant as might be thought. For pores of $1.5 \mathrm{~nm}$ diameter, the capillary condensation seems to take place in equilibrium conditions regardless of the water-wall interaction. In the wider systems with adsorption energies above $7 \mathrm{kcal} / \mathrm{mol}$ (see Figure 6 for results in the 40 A pore), the difference $\Gamma_{\text {onset }}-\Gamma_{\text {eq }}$ is hardly affected by hydrophilicity, suggesting that the hysteretic cycle in highly hydrophilic pores will not differ too much with respect to pores of moderate water affinity. This is actually what we should have expected for a pore with a strongly bound water layer, for which, as discussed above, the equilibrium and nonequilibrium surface densities become decorrelated from the water affinity of the interface. Finally, it is also interesting to note that the quantity $\Gamma_{\text {onset }}-\Gamma_{\text {eq }}$, which can be thought of as an "excess surface density" required to close the gap and cause condensation in hydrophilic pores, is strictly dependent on radius, being slightly below a monolayer in the $30 \AA$ pore and to a full monolayer, roughly $4.5 \mathrm{~nm}^{-1}$, in the $40 \AA$ pore (see Figure 6).

\section{CONCLUSIONS}

We characterized two mechanisms for the filling and surfaceadsorbed-liquid (vapor-liquid) transition in nanopores. In a material with a hydrophilic pore wall, i.e., with a water adsorption energy comparable to or higher than the water-water interaction energy, the surface density increases uniformly along the pore, until an onset filling fraction is reached and a condensed liquid phase is formed, in coexistence with the surface-adsorbed phase. This transition occurs in equilibrium in pores of $1.5 \mathrm{~nm}$ diameter but involves an overshooting of the density in larger systems. Hysteresis in adsorption-desorption isotherms can be ascribed to such an overshooting, which becomes more pronounced with increasing radius, but is approximately independent of the water-surface affinity.

On the other hand, in hydrophobic pores exhibiting adsorption energies below the interaction energy of water with itself, the rise in water content induces a localized increase of the surface density, which is seen to augment for a certain region of the pore where it forms a liquid droplet, while remaining practically constant anywhere else. As stated above, it is possible to identify a soft transition coincident with the full development of the plug, which produces a slight decrease in the density of the surfaceadsorbed phase. This is evident in Figures 3 or 4 . Therefore, an onset density $\Gamma^{*}$ onset can be defined for the hydrophobic pore, considering the average density outside the accumulation zone (we distinguish it with an asterisk from $\Gamma_{\text {onset }}$ computed from $f_{\text {onset }}$ in hydrophilic pores). Figure 6 shows, for the $4 \mathrm{~nm}$ pore, the difference between the onset density at which capillary condensation occurs ( $\Gamma_{\text {onset }}^{*}$ or $\Gamma_{\text {onset }}$ depending on hydrophilicity) and the equilibrium density when the two phases have established coexistence. This figure clearly illustrates the qualitative transition from the hydrophobic to the hydrophilic regime. According to these results, then, the phase transition in a hydrophobic porous matrix proceeds in or close to equilibrium, through a continuous development of aggregates of water molecules which grow simultaneously in the axial and radial directions. The two mechanisms discussed here can be, at a first glance, identified with those proposed by Grünberg and co-workers based on solid state NMR. ${ }^{14}$ However, a distinction must be made: these authors ascribe the difference in mechanisms to the dissimilar diameters of the pores in their samples (3.3 versus $8 \mathrm{~nm}$ silica pores) but, seemingly, always within the hydrophilic regime. The largest pores investigated in the present study are still too small to 
observe the radial filling mechanism reported in ref 14 . It can be envisioned, though, that in an hydrophilic pore of a large enough diameter, the homogeneous growth of the surface-adsorbed phase may come to an end and experience a symmetry break to continue its expansion toward the radial direction. In other words, we presume that a similar change in the mechanism of capillary condensation can result either from surface modification in pores of the same diameter or from a size increase in pores of the same hydrophilicity.

Still, a different filling mechanism should be expected for extremely hydrophobic pores, where the water affinity for the surface is three to four times smaller than in the most hydrophobic pores examined in this work. In graphitic nanopores, the water surface density is negligible until capillary condensation takes place. ${ }^{20-26}$ At variance with the pores of moderate hydrophobicity, in which an appreciable amount of water adsorbs on the surface- $-\Gamma$ may be as large as $2 \mathrm{~nm}^{-2}$ - carbon nanopores promote the aggregation of water molecules in isolated clusters of up to $1 \mathrm{~nm}$ size, associated with steep jumps and hysteresis in the adsorption isotherms. Hence, for pores on the order of $1-4 \mathrm{~nm}$ as considered in this study, three possible filling mechanims may arise: (i) a sudden condensation accompanied by pronounced hysteresis for highly hydrophobic pores; (ii) a soft, gradual filling in equilibrium, for surfaces of moderate hydrophobicity, with an affinity slightly below the one of water with itself; and (iii) an out of equilibrium transition associated with adsorption hysteresis for pores of moderate and high hydrophilicity.

Finally, we found that the equilibrium density goes up with pore size and with the water-surface affinity but converges to nearly $6 \mathrm{~nm}^{-1}$ for diameters of $30 \AA$ or more and interaction energies above $10 \mathrm{kcal} / \mathrm{mol}$. The reason behind this behavior is that, once the interface has been covered with a tightly bound layer of water molecules, the properties of the fluid become insensitive to the hydrophilicity of the solid surface. These findings show quantitative agreement with the experimental surface densities of 6.4 and $6.8 \mathrm{~nm}^{-1}$, reported at the beginning of capillary condensation in hydroxylated silica pores of $2.7 \mathrm{~nm}$ diameter. ${ }^{4}$ In the first adsorption run, when the surface is dehydroxylated, this experimental equilibrium density turns out to be $3.4 \mathrm{~nm}^{-1}$, also very close to the values emerging from our simulations in $3 \mathrm{~nm}$ diameter pores with moderate hydrophilicity (adsorption energies around $7 \mathrm{kcal} / \mathrm{mol}$, see Figure 5). A strong hysteresis is informed in ref 4 for the first adsorption run in silica nanopores, which should not be interpreted as contradictory to our results: in the experiment, the pronounced hysteresis in the first cycle is not a consequence of hydrophobicity but is caused by a dramatic change in the nature of the surface between adsorption and desorption.

\section{AUTHOR INFORMATION}

\section{Corresponding Authors}

*E-mail: valeria.molinero@utah.edu (V.M.); damian@qi.fcen. uba.ar (D.A.S.).

\section{ACKNOWLEDGMENT}

This work has been supported by collaborative research grant PICT 2007-2111 of the Agencia Nacional de Promoción Científica y Tecnológica de Argentina (V.M. and D.A.S.). E.d.l.L. acknowledges CONICET for a doctoral fellowship. We thank the Center of High Performance Computing at the University of Utah for allocation of computer time.

\section{REFERENCES}

(1) Ng, E.-P.; Mintova, S. Microporous Mesoporous Mater. 2008, $114,1$.

(2) Papadopoulou, A.; van Swol, F. J. Chem. Phys. 1992, 97, 6942.

(3) Branton, P. J.; Hall, P. G.; Treguer, M.; Sing, K. S. W. J. Chem. Soc., Faraday Trans. 1995, 91, 2041.

(4) Inagaki, S.; Fukushima, Y. Microporous Mesoporous Mater. 1998, $21,667$.

(5) Vishnyakov, A.; Neimark, A. J. Phys. Chem. B 2001, 105, 7009.

(6) Matsumoto, A.; Sasaki, T.; Nishimiya, N.; Tsutsumi, K. Langmuir 2001, 17, 47.

(7) Thommes, M; Smarsly, B; Groenewolt, M; Ravikovitch, P. I.; Neimark, A. V. Langmuir 2006, 22, 756.

(8) Kocherbitov, V.; Alfredsson, V. J. Phys. Chem. C 2007, 111, 12906.

(9) de la Llave, E.; Molinero, V.; Scherlis, D. A. J. Chem. Phys. 2010, $133,34513$.

(10) Morishige, K.; Yoshida, K. J. Phys. Chem. B 2011, 114, 7095.

(11) Kocherbitov, V.; Alfredsson, V. Langmuir 2011, 27, 3889.

(12) Llewellyn, P. L.; Schueth, F.; Grillet, Y.; Rouquerol, F.; Rouquerol, J.; Unger, K. K. Langmuir 1995, 11, 574.

(13) Takahara, S.; Nakano, M.; Kittaka, S.; Kuroda, Y.; Mori, T.; Hamano, H.; Yamaguchi, T. J. Phys. Chem. B 1999, 103, 5814.

(14) Grünberg, B.; Emmler, T.; Gedat, E.; Shenderovich, I.; Findenegg, G. H.; Limbach, H.-H.; Buntkowsky, G. Chem.-Eur. J. 2004, 10, 5689.

(15) Llewellyn, P.; Grillet, Y.; Schüth, F.; Reichert, H. Microporous Mater. 1994, 3, 345.

(16) Branton, P. J.; Hall, P. G.; Sing, K. S. W.; Reichert, H.; Schuth, F. S.; Unger, K. K. J. Chem. Soc., Faraday Trans. 1994, 90, 2965.

(17) Ravikovitch, P. I.; O Domhnaill, S. C.; Neimark, A. V.; Schüth, F.; Unger, K. K. Langmuir 1995, 11, 4765.

(18) Hummer, G.; Rasaiah, J. C.; Noworyta, J. P. Nature 2001, 414, 188.

(19) Do, D. D.; Do, H. D. Carbon 2000, 38, 767.

(20) Striolo, A.; Gubbins, K. E.; Gruszkiewicz, M. S.; Cole, D. R.; Simonson, J. M.; Chialvo, A. A.; Cummings, P. T.; Burchell, T. D.; More, K. L. Langmuir 2005, 21, 9457.

(21) Striolo, A.; Chialvo, A. A.; Cummings, P. T.; Gubbins, K. E. J. Chem. Phys. 2006, 124, 74710.

(22) Iiyama, T.; Ruike, M.; Kaneko, K. Chem. Phys. Lett. 2000, $331,359$.

(23) Ohba, T.; Kanoh, H.; Kaneko, K. J. Phys. Chem. B 2004, $108,14964$.

(24) Ohba, T.; Kanoh, H.; Kaneko, K. J. Am. Chem. Soc. 2004, 126,1560 .

(25) Ohba, T.; Kanoh, H.; Kaneko, K. Nano Lett. 2005, 5, 227.

(26) Ohba, T.; Kanoh, H.; Kaneko, K. Chem.-Eur. J. 2005, 11, 4890 .

(27) Tao, Y.; Endo, M.; Kaneko, K. J. Am. Chem. Soc. 2009, 131, 904.

(28) Tao, Y.; Endo, M.; Kaneko, K. J. Am. Chem. Soc. 2010, 132, 1214.

(29) Shirono, K.; Daiguji, H. J. Phys. Chem. C 2007, 111, 7938

(30) Takahara, S.; Sumiyama, N.; Kittaka, S.; Yamaguchi, T.; BellissentFunel, M.-C. J. Phys. Chem. B 2005, 109, 11231.

(31) Romero-Vargas Castrillón, S.; Giovambattista, N.; Aksay, I. A.; Debenedetti, P. G. J. Phys. Chem. B 2009, 113, 7973.

(32) Romero-Vargas Castrillón, S.; Giovambattista, N.; Aksay, I. A.; Debenedetti, P. G. J. Phys. Chem. B 2009, 113, 1438.

(33) Saugey, A.; Bocquet, L.; Barrat, J. L. J. Phys. Chem. B 2005, 109,6520 .

(34) Monson, P. A. Langmuir 2008, 24, 12295.

(35) Brovchenko, I.; Geiger, A.; Oleinikova, A. J. Chem. Phys. 2004, 120, 1958.

(36) Chakraborty, A.; Baran Saha, B.; Koyama, S. J. Chem. Eng. Data 2009, 54, 448 . 
(37) Siboulet, B.; Coasne, B.; Dufrêche, J.-F.; Turq, P. J. Phys. Chem. B 2011, 115, 7881.

(38) Molinero, V.; Moore, E. B. J. Phys. Chem. B 2009, 113, 4008.

(39) Stillinger, F. H.; Weber, T. A. Phys. Rev. B 1985, 31, 5262.

(40) Moore, E. B.; Molinero, V. J. Chem. Phys. 2009, 130, 244505.

(41) Moore, E. B.; de la Llave, E.; Welke, K.; Scherlis, D. A.; Molinero, V. Phys. Chem. Chem. Phys. 2010, 12, 4124.

(42) Kastelowitz, N.; Johntson, J. C.; Molinero, V. J. Chem. Phys. 2010, 132, 124511.

(43) Jacobson, L. C.; Hujo, W.; Molinero, V. J. Phys. Chem. B 2009, 113, 10298.

(44) Plimpton, S. J. J. Comput. Phys. 1995, 117, 1. 